\title{
Adherencia y cumplimiento de las recomendaciones de cuidado y prevención del pie diabético por parte de médicos tratantes en dos hospitales de Lima, Perú Physicians' adherence and compliance with recommendations for care and prevention of diabetic foot, in two hospitals from Lima, Peru
}

Correspondencia Germán Málaga Rodríguez german.malaga@upch.pe

Recibido: 07/05/2017

Arbitrado por pares

Aprobado: 19/07/2017

Citar como: Aphang M, Lazo-Porras $M$, Beltrán-Ale G, CardenasMontero D, Vera R, Málaga G. Adherencia y cumplimiento de las recomendaciones de cuidado y prevención del pié diabético por parte de médicos tratantes en dos hospitales de Lima, Perú. Acta Med Peru. 2017;34(3):168-72

\author{
Meylin Aphang 1,2, María Lazo-Porras ${ }^{3,4}$, Guillermo Beltrán-Ale ${ }^{3}$, Deborah \\ Cardenas-Montero ${ }^{3}$, Rosialzira Vera ${ }^{5}$, Germán Málaga ${ }^{1,3,6}$ \\ 1 Facultad de Medicina Alberto Hurtado, Universidad Peruana Cayetano Heredia. Lima, Perú. \\ 2 Servicio de Medicina Interna, Hospital Arzobispo Loayza. Lima, Perú. \\ 3 CONEVID Unidad de Conocimiento y evidencia, Universidad Peruana Cayetano Heredia. Lima, Perú. \\ 4 CRONICAS Centro de Excelencia en Enfermedades Crónicas, Universidad Peruana Cayetano Heredia. Lima, Perú. \\ 5 Hospital Universitario Fundación Jimenes Diaz. Madrid, España. \\ 6 Servicio de Medicina Interna, Hospital Cayetano Heredia. Lima, Perú
}

\section{RESUMEN}

Objetivo: Comparar el cumplimiento por parte de los médicos de las recomendaciones que establecen las guías de manejo y prevención de pie diabético entre un hospital público y uno privado. Materiales y métodos: Realizamos un análisis secundario de la base de datos del estudio transversal "Calidad del control metabólico en pacientes ambulatorios con diabetes mellitus tipo 2 atendidos en dos hospitales generales de Lima". Se utilizaron dos cuestionarios, el primero para recolectar datos demográficos y un segundo de 5 preguntas aplicados al terminar su cita en el consultorio externo de endocrinología: 1) Pedido del médico de retirarse los zapatos, 2) Evaluación de sensibilidad en extremidades inferiores, 3) Recomendación de zapatos especiales, 4) Derivación al servicio de pie diabético y 5) Uso de zapatos especiales por el paciente. Para el análisis de datos se calculó la proporción y los intervalos de confianza al 95\% del cumplimiento de las recomendaciones y se usó chi cuadrado o la prueba exacta de Fisher para evaluar asociación entre tipo de hospital y cumplimiento de las recomendaciones. Resultados: Se encontró una diferencia significativa entre el hospital público y el hospital privado $(p<0,05)$ en el cumplimiento de cuatro de las cinco recomendaciones. En los cuatro casos el cumplimiento fue mayor en el hospital privado que en el hospital público. Las recomendaciones con diferencia significativa fueron: 1. Participante afirmó haberse retirado los zapatos por pedido del médico ( $37 \%$ hospital público vs $77 \%$ hospital privado $p<0,001)$; 2 . Paciente recibió recomendación de usar calzado especial ( $16 \%$ hospital público vs $61 \%$ hospital privado $p<0,001$ ) 3. Paciente fue derivado a un servicio de pie diabético ( $3 \%$ hospital público vs $18 \%$ hospital privado $p=0,001)$; 4 . Paciente afirmaba usar calzado especial ( $64 \%$ hospital público vs $82 \%$ hospital privado $p=0,012$ ). La única recomendación que no tuvo una diferencia significativa fue el examen de búsqueda de neuropatía (45\% hospital público vs $54 \%$ hospital privado). Conclusiones: El cumplimiento de las recomendaciones por parte de los médicos de cuidado de pies en pacientes con Diabetes mellitus tipo 2, fue significativamente mayor en la institución privada vs. La pública. Sin embargo, incluso en la institución privada el resultado distó de ser idóneo. La mejor performance en el hospital privado, sugiere que existirían diferencias de la calidad de la atención, lo que tendría repercusiones en la salud de las personas.

Palabras clave: Pie diabético; Diabetes Mellitus tipo 2; Encuestas de atención de la salud; Adhesión a directriz; Calidad de la atención de salud (fuente: DeCS BIREME). 


\section{ABSTRACT}

Objective: To compare physicians' compliance with the recommendations established by the guides for diabetic foot management and prevention in a public hospital and a private hospital. Materials and methods:: This is a secondary analysis study of the data base from the 'Quality of Metabolic Control in Type 2 Diabetes Outpatients Managed in Lima General Hospitals'. Two questionnaires were used, the first one for collecting demographic data, and the second with 5 questions, and both were administered at the end of their appointment in the endocrinology clinic. The second questionnaire requested the following information: i. Request from the physician to take out their shoes. ii. Sensitivity assessment in the legs. iii. Recommending the use of special shoes. iv. Referring patients to the diabetic foot service, and v. Use of special shoes by the patients. The rates and the $95 \%$ confidence intervals for compliance with the aforementioned recommendations were calculated, and chisquare test and Fisher's exact method were used for determining an association between the hospital type and compliance with the recommendations. Results: A significant difference was found between the public hospital and the private hospital $(p<0.05)$ in complying with four of five recommendations (in these four cases compliance was higher in the private hospital). These were as follows: 1 . The participants declared having taken out their shoes at the physicians' request (37\% public vs. $77 \%$ private, $p<0.001) ; 2$. The patients received a recommendation about using special shoes $(16 \%$ public vs. $61 \%$ private, $\mathrm{p}<0.001$ ); 3 . Patients were referred to a diabetic foot service (3\% public vs. $18 \%$ private, $p=0.001$ ); 4 . Patients reported using special shoes (64\% public vs. $82 \%$ private, $p=0.012$ ). Conclusions: Compliance with physicians' recommendations regarding feet care in patients with type 2 diabetes mellitus was significantly higher in a private hospital compared to a public hospital. However, even in the private institution the result was far from being optimal. The best performance in the private hospital suggests there are differences in quality of care, which may have consequences in people's health.

Keywords: Diabetic foot; Diabetes Mellitus type-2; Health care surveys; Guideline adherence; Quality of health care (source: MeSH NLM).

\section{INTRODUCCIÓN}

En países en vías de desarrollo suele existir una marcada diferencia en la calidad de atención recibida por las personas, según el nivel socio económicas del que proceden. En el Perú, la población de menores recursos socioeconómicos suele recibir atención en hospitales públicos mientras que los pacientes con mayores recursos pueden acudir hospitales privados con diferencias considerables en costos de atención ${ }^{[1]}$.

Estudios realizados en países con condiciones socioeconómicas similares a las del Perú muestran que tanto en las instituciones públicas como las privadas existe falta de adherencia del personal de salud a recomendaciones de guías clínicas ${ }^{[2-4]}$. Usualmente hay una tendencia al exceso de intervenciones en el sector privado y la carencia de las mismas en el público, además existe mayor morbimortalidad en el sector público que generalmente incluye pacientes con menores recursos económicos, de mayor severidad, con atención más tardía y probablemente de menor calidad ${ }^{[1]}$.

Con la intención de objetivizar estas diferencias realizamos un análisis secundario de una base de datos que contenía información acerca del cumplimiento por parte del personal de salud de recomendaciones de guías clínicas en un hospital público y uno privado. Específicamente guías para la prevención y manejo del pie diabético.

Según las guías de prevención y manejo de pie diabético, incluyendo la guía del Ministerio de Salud, anualmente se debe realizar una evaluación al paciente que incluya la verificación de deformidades, pruebas para detectar neuropatía y evaluación vascular; en base a esta evaluación se puede estratificar al paciente de acuerdo a su riesgo de ulceración y determinar el manejo individualizado de cada paciente. Es sabido que una estrategia que incluya: "prevención, educación al paciente y el tratamiento multidisciplinario de las úlceras" puede reducir las tasas de amputación y mejora la sobrevida de los pacientes de manera costo efectiva ${ }^{[5-8]}$.

Por ello, el objetivo principal de este estudio es determinar si existen diferencias en el cumplimiento por parte del personal de salud de las recomendaciones que establecen las guías de manejo y prevención de pie diabético, entre un hospital público y uno privado.

\section{MATERIALES Y MÉTODOS}

\section{Participantes y diseño del estudio}

Se realizó un análisis secundario de la base de datos del estudio transversal "Calidad del control metabólico en pacientes ambulatorios con diabetes mellitus tipo 2 atendidos en dos hospitales generales de Lima". Los hospitales incluidos en este estudio se encuentran contiguos en el distrito de San Martin de Porres siendo uno de estos, público y el otro privado. El periodo de reclutamiento en el hospital público fue en el período de marzo - julio del 2014, mientras que, en el hospital privado, el reclutamiento fue de julio 2014 a diciembre del 2015 . El muestreo fue aleatorio. Los criterios de inclusión fueron: pacientes con diagnóstico $>1$ año de DM2 según criterios de la ADA ${ }^{[8]}$, excluyéndose a pacientes hospitalizados en el último año por complicaciones agudas de la enfermedad, a aquellos con diagnóstico de DM secundaria ${ }^{[8]}$, quienes padecieran alguna 
Tabla 1. Preguntas de evaluación de cuidado y prevención.

\begin{tabular}{cl} 
Item & \multicolumn{1}{c}{ Pregunta } \\
\hline 1 & $\begin{array}{l}\text { Pedido del médico de retirarse los zapatos durante la } \\
\text { consulta. }\end{array}$ \\
2 & $\begin{array}{l}\text { Evaluación de sensibilidad en extremidades inferiores } \\
\text { por el médico }\end{array}$ \\
3 & Recomendación de zapatos especiales por el médico \\
4 & Derivación al servicio de pie diabético por el médico \\
5 & Uso de zapatos especiales por el paciente \\
\hline
\end{tabular}

enfermedad crónica no metabólica y a quienes no firmaron el consentimiento informado.

\section{Procedimientos}

Los participantes fueron reclutados al salir de la consulta externa del Servicio de Endocrinología de cada uno de los hospitales. Se aplicó un cuestionario para recolectar información de características socio-demográficas, estilos de vida y relacionadas a la historia de enfermedad, además de un cuestionario con 5 preguntas relacionadas con el cumplimiento por parte del médico, con las recomendaciones para la evaluación de pie diabético. Además, a todos los participantes se les tomó una muestra de sangre para el análisis de hemoglobina glicosilada.

\section{Variables de estudio}

Como variables principales se tomó el cumplimiento de las recomendaciones de la evaluación del pie diabético brindadas por la American Diabetes Association (ADA) ${ }^{[8]}$. Estas cinco recomendaciones están incluidas en la Tabla 1. La variable exposición fue el tipo de hospital: el Hospital Nacional Cayetano Heredia como un hospital público y la Clínica Médica Cayetano Heredia como hospital privado. Como variables secundarias se consideraron el sexo, la edad, los años de diagnóstico de diabetes ( $<10$ años, >=10 años), el índice de masa corporal (IMC) de los participantes considerado como normal $\left(18,5-<25 \mathrm{~kg} / \mathrm{m}^{2}\right)$, sobrepeso $\left(\geq 25,0-<30 \mathrm{~kg} / \mathrm{m}^{2}\right)$ y obesidad $(\geq 30)$ y hemoglobina glicosilada.

\section{Plan de análisis}

Se describieron las características generales de los participantes de cada grupo. Se utilizó frecuencias para variables cualitativas, y medias y desviación estándar para variables cuantitativas.

Cada pregunta relacionada al cumplimiento de las recomendaciones fue analizada por separado, obteniendo la proporción de respuestas afirmativas en cada grupo y los intervalos de confianza (IC) al 95\%. Además, se evaluó si existía asociación entre cada una de las preguntas evaluadas y el tipo de hospital mediante la prueba de chi cuadrado. En caso de frecuencias esperadas menores a $5 \%$ se utilizó la prueba de Fisher. Por tratarse de un análisis de base de datos secundaria se calculó el poder estadístico usando el programa STATA 12 utilizando una diferencia de proporciones de $13 \%$ según lo encontrado en el estudio de adherencia a guías de práctica clínica en Obstetricia en la India entre hospitales públicos y privados ${ }^{[9]}$. El poder encontrado para las asociaciones de interés es de $85 \%$.

\section{Aspectos Éticos}

El presente es un análisis secundario de datos que fue aprobado por los comités de ética de la Universidad Peruana Cayetano Heredia y del Hospital Nacional Cayetano Heredia y la autorización de la Clínica Médica Cayetano Heredia. Para el presente estudio no se tuvo contacto alguno con sujetos humanos. En tal sentido, los posibles riesgos para los sujetos del análisis son mínimos. La información manejada para este estudio fue codificada para respetar la confidencialidad de los participantes.

\section{RESULTADOS}

Se revisaron entrevistas hechas de manera aleatorizada a 123 participantes del hospital público y 60 participantes del hospital privado.

Las características de poblaciones del hospital público y privado se muestran en la Tabla 2, en el hospital público de los participantes $65,8 \%$ fueron mujeres, mientras que en el hospital privado fue de 52,6\%. El tiempo de enfermedad fue de 10,2 años (DE 9) en el hospital público y de 8,1 años (DE 6) en el hospital privado.

Se encontró una diferencia significativa entre el hospital público y el hospital privado $(p<0,05)$ en el cumplimiento de cuatro de las cinco recomendaciones. En los cuatro casos el cumplimiento fue mayor en el hospital privado que en el hospital público. Las recomendaciones con diferencia significativa fueron: 1)

Tabla 2. Características generales de los pacientes.

\begin{tabular}{lcc} 
& $\begin{array}{c}\text { Hospital } \\
\text { público } \\
(\mathbf{N}=\mathbf{1 2 3}) \\
\mathbf{n}(\%)\end{array}$ & $\begin{array}{c}\text { Hospital } \\
\text { privado } \\
(\mathbf{N}=\mathbf{5 7}) \\
\mathbf{n}(\%)\end{array}$ \\
\hline Edad, media (DE) & $61,8(11,1)$ & $63,1(13,2)$ \\
Mujeres & $81(65,8 \%)$ & $32(52,6 \%)$ \\
Años de diagnóstico, media (DE) & $10,2 \pm 9$ & $8,1 \pm(6)$ \\
Tiempo de enfermedad & & \\
$\quad$ Menos de 10 años & $61(49,59 \%)$ & $18(30,0 \%)$ \\
$\quad$ Más de 10 años & $62(50,41 \%)$ & $42(70,0 \%)$ \\
Índice de masa corporal & & \\
\multicolumn{1}{|c|}{ Normal } & $23(18,7 \%)$ & $23(38,3 \%)$ \\
$\quad$ Sobrepeso & $56(45,5 \%)$ & $35(21,0 \%)$ \\
$\quad$ Obesidad & $44(36,3 \%)$ & $16(26,7 \%)$ \\
Hemoglobina glicosilada & $8,2 \pm 2,2$ & $7,7(2,0)$ \\
\hline
\end{tabular}

* DE: Desviación estándar 
Tabla 3. Proporción de pacientes que respondieron afirmativamente según pregunta.

\begin{tabular}{cccccc} 
& \multicolumn{2}{c}{$\begin{array}{c}\text { Hospital público } \\
\mathbf{n ( \% )}\end{array}$} & \multicolumn{2}{c}{$\begin{array}{c}\text { Hospital privado } \\
\mathbf{n}(\%)\end{array}$} & Chi cuadrado \\
\cline { 2 - 6 } & Sí & IC 95\% & Sí & IC 95\% & p \\
\hline 1 & $45(36,6 \%)$ & $29-46 \%$ & $46(76,7 \%)$ & $65-86 \%$ & $<0,001$ \\
2 & $55(44,7 \%)$ & $36-54 \%$ & $32(53,3 \%)$ & $42-67 \%$ & 0,113 \\
3 & $20(16,2 \%)$ & $10-23 \%$ & $37(61,7 \%)$ & $48-73 \%$ & $<0,001$ \\
4 & $4(3,3 \%)$ & $1-8 \%$ & $11(18,3 \%)$ & $10-29 \%$ & 0,001 (Fisher) \\
5 & $79(64,2 \%)$ & $55-72 \%$ & $49(81,7 \%)$ & $71-90 \%$ & 0,012 \\
\hline
\end{tabular}

Participante afirmó haberse retirado los zapatos por pedido del médico (37\% hospital público vs $77 \%$ hospital privado $p<0,001$ ); 2) Paciente recibió recomendación de usar calzado especial ( $16 \%$ hospital público vs $61 \%$ hospital privado $p<0,001) 3$ ) Paciente fue derivado a un servicio de pie diabético ( $3 \%$ hospital público vs $18 \%$ hospital privado $p=0,001)$; 4) Paciente afirmaba usar calzado especial ( $64 \%$ hospital público vs $82 \%$ hospital privado $p=0,012)$. La única recomendación que no tuvo una diferencia significativa fue el examen de búsqueda de neuropatía (45\% hospital público vs $54 \%$ hospital privado) (Tabla 3 ).

\section{DISCUSIÓN}

En nuestro estudio hallamos mayor cumplimiento de las recomendaciones que establecen las guías de manejo y prevención de pie diabético en el hospital privado comparado con el público. Asimismo, hallamos que la evaluación recomendada del pie de pacientes con diabetes mellitus 2 de acuerdo a las mismas recomendaciones, es deficiente. Aunque el resultado fue mejor en el ambiente privado, en ninguno fue óptimo.

En nuestro país no se ha explorado la adherencia del médico a las guías de prevención y manejo del pie diabético y este estudio resulta una primera aproximación para conocerla.

Los resultados mostrados son insatisfactorios y deberían ser difundidos, analizados y discutidos, así como explorar en nuevos estudios, sobre cuáles son las barreras que permitan identificar medidas de mejora en la calidad de atención de los pacientes. Entre las posibles explicaciones a las deficiencias encontradas, tenemos por un lado en el sector público: la limitación de recursos, las limitaciones de infraestructura, el limitado espacio, las interrupciones que ocurren en las consultas, el tiempo dedicado para la atención de cada paciente, la carencia de instrumental, como monofilamento o diapasón, que permita completar el examen físico del paciente ${ }^{(1)}$. Mientras que en el caso de los pacientes que son atendidos en el sector privado se podría plantear que se trate de factores asociados a la sobrecarga de los escasos endocrinólogos que existen el País ${ }^{[10]}$ pero en ambos casos, la ausencia de un sistema de atención primaria que permita sostener la mayoría de consultas antes de que lleguen en busca de una atención especializada debe ser un factor a considerar ${ }^{[1,11]}$.

Diversos estudios sobre la calidad de la atención en entidades de salud públicas en nuestro país muestran diferentes grados de insatisfacción de los usuarios, cuyas causas se originan por diferentes factores como tiempo de espera largo, calidad de la atención del personal médico y no médico, servicios complementarios como laboratorio, procedimientos de ayuda diagnóstica, farmacia, etc., a diferencia de la calidad del servicio recibido en instituciones privadas, donde la satisfacción del usuario, suele ser mayor ${ }^{[1,12]}$.

El aspecto más bajo, fue el relacionado con la derivación a un servicio de pie diabético, situación no relacionada necesariamente con deficiente cumplimiento de recomendaciones, sino por la circunstancia de no contar con atención especializada, aun cuando el beneficio que este tipo de servicios provee, en términos de prevención de úlceras, de amputaciones y de mortalidad, está ampliamente demostrado ${ }^{[7,11]}$.

Incluso una recomendación que probablemente no requiere mayor infraestructura o equipamiento para su ejecución como es la recomendación del uso de calzado especial, mostró una marcada diferencia, esto se debería a que los médicos en el sector público no cuentan con la misma cantidad de tiempo que puedan invertir en educación por cada paciente ${ }^{[1]}$ respecto al que cuentan en el sector privado o asumen que su coste no podrá ser solventado y por tanto priorizan otros aspectos en la consulta.

\section{Comparación con otros estudios}

La adherencia a las recomendaciones brindadas por las guías de prevención y manejo de pie diabético es en general baja. En Pakistán, 30\% de pacientes seguidos durante más de 8 años, fueron informados sobre complicaciones derivadas de pie diabético, mientras que, en Marruecos, sólo el $18 \%$ tuvo alguna evaluación relacionada a lo largo de su enfermedad ${ }^{[13]}$. Sin embargo, esta situación no llega a ser óptima ni siquiera en sistemas de salud avanzados, por ejemplo en un estudio español diseñado para evaluar el impacto de una intervención educativa para mejorar la evaluación médica a los pacientes diabéticos, 
evidenció que sólo el 19,5\% de los médicos examinó los pies de los pacientes, situación que mejoró, discretamente, después de la intervención a $30 \%{ }^{[14]}$ o como se pudo apreciar en un estudio en hispanos en USA, el $72 \%$ tuvo evaluación de pies, pero en caso de prescripción de zapatos especiales para personas con diabetes mellitus 2 , sólo son utilizados de acuerdo con las recomendaciones por el 22 a $28 \%$ de ellos ${ }^{[15]}$.

\section{Importancia en salud pública}

La DM 2 es un problema de salud pública por su prevalencia y los costos que demanda su manejo, especialmente el de sus complicaciones ${ }^{[5,7]}$, habiéndose evidenciado en un estudio de Vigilancia epidemiológica nacional, en el 2012, que uno de cada tres diabéticos presentaba alguna complicación crónica siendo las más frecuentes la neuropatía, el pie diabético y la insuficiencia renal ${ }^{[16]}$.

Las complicaciones del pie diabético acumulan más hospitalizaciones que cualquier otra complicación de la DM2 con mortalidad y morbilidad considerables. Asimismo, son las más serias y costosas de esta enfermedad ${ }^{[5,7]}$. Estrategias que incluyen prevención, educación de pacientes y prestadores de salud, tratamiento multidisciplinario de las úlceras y monitoreo estrecho reducen las amputaciones entre 49 y $85 \%$ [5,7,8,17].

Cabe resaltar que la investigación en cuidados del pie de pacientes diabéticos y la implementación de programas de prevención es una de las formas más costo efectivas de provisión de cuidados de salud ${ }^{[18]}$.

\section{Limitaciones}

Se trató de un análisis secundario de datos, con un objetivo principal de estudio diferente. Si bien, la cantidad de participantes especialmente en el hospital privado es pequeña, contamos con un poder estadístico superior al $80 \%$ para sustentar las diferencias planteadas.

Para conocer el cumplimiento de la evaluación de pie diabético se utilizó sólo la entrevista al paciente y no filmación de las consultas médicas para verificar el cumplimiento de las recomendaciones.

En conclusión, la adherencia a las recomendaciones de cuidado de pies en pacientes con DM2, es muy deficiente, auque, fue significativamente mejor en la institución privada vs. La pública.

Este estudio pone de manifiesto la necesidad de implementar intervenciones dirigidas a médicos para que se adhieran a las guías de práctica clínica existentes y aceptadas internacionalmente sobre el cuidado y vigilancia de los pies del paciente diabético y a los pacientes, para que se empoderen en su cuidado y de esta manera se contribuya a prevenir la inmensa carga que representa esta temible complicación, tanto para el paciente como para el sistema de salud, con la consiguiente mejora en su calidad de vida e impacto socioeconómico positivo que en términos de costo-utilidad y costo-beneficio indican ser altamente favorables.

\section{REFERENCIAS BIBLIOGRÁFICAS}

1. Alcalde-Rabanal JE, Lazo-González O, Nigenda G. Sistema de salud de Perú. Salud Publica Mex. 2011;53 supl 2:S243-54.

2. Ferreira GM, Correia LC, Reis H, Ferreira CB, Freitas F, Ferreira M, et al. Maior letalidade e morbidade por infarto agudo do miocárdio em hospital público, Em Feira de Santana - Bahia. Arq Bras Cardiol. 2009:93(2):97-104

3. Nogueira L, de Sousa RM, Padilha K, Koike K. Características clínicas e gravidade de pacientes internados em UTIs públicas e privadas. Texto Contexto - Enferm. 2012:21(1):59-67.

4. Macinko J, Harris MJ. Brazil's family health strategy - delivering community-based primary care in a universal health system. N Engl J Med. 2015;372(23):2177-81.

5. Servicio de Endocrinología, Hospital Sabogal. Manual para el diagnóstico y tratamiento del pie diabético. Lima; Merck \& Co., Inc.; 2014.

6. Bus SA, Armstrong DG, van Deursen RW, Lewis JE, Caravaggi CF, Cavanagh PR. International Working Group on the Diabetic Foot IWGDF guidance on footwear and offloading interventions to prevent and heal foot ulcers in patients with diabetes. Diabetes Metab Res Rev. 2016;32 Suppl 1:25-36.

7. Stockl K, Vanderplas A, Tafesse E, Chang E. Costs of lower-extremity ulcers among patients with diabetes. Diabetes Care. 2004;27(9):212934.

8. American Diabetes Association. Standards of medical care in diabetes 2014. Diabetes Care. 2014;37 Suppl 1:S14-S80.

9. Nagpal J, Sachdeva A, Sengupta Dhar R, Bhargava VL, Bhartia A. Widespread non-adherence to evidence-based maternity care guidelines: a population-based cluster randomised household survey. BJOG. 2015;122(2):238-47.

10. Solo hay diez endocrinólogos por cada millón de habitantes en el Perú [Internet]. Lima: Diario Médico; 2017 [citado el 25 de abril de 2017]. Disponible en: http://www.diariomedico.pe/?p=7851

11. Anticona Huaynate CF, Pajuelo Travezaño MJ, Correa M, Mayta Malpartida H, Oberhelman R, Murphy LL, et al. Diagnostics barriers and innovations in rural areas: insights from junior medical doctors on the frontlines of rural care in Peru. BMC Health Serv Res. 2015;15:454.

12. Casalino-Carpio GE. Calidad de servicio de la consulta externa de Medicina Interna de un hospital general de Lima mediante la encuesta Servqual. Rev Soc Peru Med Interna. 2008;21(4):143-52.

13. Sheikh MA, Hakeem R, Asar F, Shaikh AH. Diabetes education and care in a developing country: observations from Karachi, Pakistan. Prim Care Diabetes. 2015;9(1):48-53.

14. Vidal-Pardo J, Perez-Castro T, Lopez-Alvarez X, Santiago-Perez M, Garcia Soidan F, Muniz J. Effect of an educational intervention in primary care physicians on the compliance of indicators of good clinical practice in the treatment of type 2 diabetes mellitus [OBTEDIGA project]. Int J Clin Pract. 2013;67(8):750-8.

15. Welch G, Allen NA, Zagarins SE, Stamp KD, Bursell SE, Kedziora RJ. Comprehensive diabetes management program for poorly controlled Hispanic type 2 patients at a community health center. Diabetes Educ. 2011;37(5):680-8.

16. Ramos W, López T, Revilla L, More L, Huamaní M, Pozo M. Resultados de la vigilancia epidemiológica de diabetes mellitus en hospitales notificantes del Perú, 2012. Rev Peru Med Exp Salud Publica. 2014;31(1):9-15

17. Bus SA, Waaijman R, Nollet F. New monitoring technology to objectively assess adherence to prescribed footwear and assistive devices during ambulatory activity. Arch Phys Med Rehabil. 2012;93(11):2075-9.

18. Seguel G. ¿Por qué debemos preocuparnos del pie diabético? Importancia del pie diabético. Rev Med Chile. 2013;141(11):1464-9. 